\title{
Under or overpressure: an audit of endotracheal cuff pressure monitoring at the tertiary care center
}

\author{
Biju Viswambharan ${ }^{1}$, Manjini Jeyaram Kumari', Gopala Krishnan ${ }^{2}$, Lakshmi Ramamoorthy ${ }^{1}$ \\ ${ }^{1}$ Department of Medical Surgical Nursing, College of Nursing and ${ }^{2}$ Department of Neurosurgery, Jawaharlal Institute of Postgraduate Medical Education and \\ Research, Puducherry, India
}

Background: Mechanical ventilation is a lifesaving intervention for critically ill patients but can produce the major complication of ventilator-associated pneumonia (VAP). Inappropriately inflated endotracheal tubes cause potential harm due to high or low pressure; this can be prevented through monitoring protocols.

Methods: A cross-sectional study of 348 cuff pressure readings was performed with intubated and mechanically ventilated patients to evaluate the exact proportion of patients in intensive care units (ICUs) where the cuff pressure is optimal and to identify the ICUs where device-based monitoring is available to produce a lower proportion of sub-optimal cuff pressure cases. Every three days, cuff pressure was assessed with a handheld cuff pressure manometer. The corresponding VAP rates of those ICUs were obtained from the hospital infection control department.

Results: Cuff pressure of $40.2 \%$ was the lower cutoff for the high category, that of optimal was $35.3 \%$, and the highest cutoff of sub-optimal was $24.4 \%$. This study also showed ICUs that had cuff pressure monitoring devices and protocols. Active measurement protocols had a higher proportion of optimal cuff pressure (58.5\%) and a lower proportion of sub-optimal and high cuff pressure (19.5\% and 22.0\%) compared to ICUs with no device-based monitoring protocols. Furthermore, the VAP rate of ICUs exhibited a weak positive correlation with sub-optimal cuff pressure.

Conclusions: Device-based cuff pressure monitoring is essential in maintaining adequate cuff pressure but often is inadequate, resulting in high readings. Therefore, this study suggests that device-based cuff pressure monitoring be practiced.

Key Words: airway management; artificial respiration; endotracheal; hospital acquired pneumonia; intubation; mechanical ventilation; ventilator-associated pneumonia

\section{INTRODUCTION}

Mechanical ventilation is a lifesaving intervention for critically ill patients. Even though this maneuver can help critically ill patients with associated risk factors, it contributes to difficult treatment process and weaning. Among the 427 health care-associated infections observed, pneumonia was the most common, and $32 \%$ of those cases were ventilator-associated [1]. A large number of patients receiving mechanical ventilation are initially treated with an endo-tracheal tube as a dependable entry point to the airway. The end of this connecting tube is

\section{Original Article}

Received: January 4, 2021

Revised: July 7, 2021

Accepted: August 18, 2021

Corresponding author

Manjini Jeyaram Kumari Department of Medical Surgical Nursing, College of Nursing, Jawaharlal Institute of Postgraduate Medical Education and Research, Puducherry 605006, India Tel: +98-9459-5420

E-mail:kumari_mj@ymail.com

Copyright (C) 2021 The Korean Society of Critical Care Medicine

This is an Open Access article distributed under the terms of Creative Attributions Non-Commercial License (https:// creativecommons.org/li-censes/by-nc/4.0/) which permits unrestricted noncommercial use, distribution, and reproduction in any medium, provided the original work is properly cited. 
attached to an air-filled balloon and positioned snuggly in the trachea to prevent air leaks and aspiration [2,3]. The air-filled balloon often is called an endo-tracheal cuff, and the pressure is referred to as endo-tracheal cuff pressure and must be in a safe range of $20-30 \mathrm{~cm} \mathrm{H}_{2} \mathrm{O}$ to ensure delivery of ordered mechanical ventilation and tidal volume. Proper pressure can decrease the risk for aspiration of secretions that accumulate above the cuff and maintains adequate tracheal perfusion. There is no acceptable practice for measuring cuff pressure even though it has been shown that a cuff pressure below 20 cm $\mathrm{H}_{2} \mathrm{O}$ contributes to a fourfold risk of occurrence of ventilator-associated pneumonia (VAP) among the critically ill [2-6].

There are various elements contributing to VAP from which cuff pressure monitoring can be performed by registered nurses and can have help prevent VAP [7]. However, there are no direct studies to establish the importance of nurse-led cuff pressure monitoring and its impact on VAP prevention. Therefore, the study aim was to determine the exact proportion of patients in intensive care units (ICUs) with optimal cuff pressure, identify the ICUs where device-based monitoring is available to achieved a lower proportion of patients with sub-optimal cuff pressure, and to find the correlation of VAP rate in ICUs with the proportion of patients who exhibited optimal cuff pressure.

\section{MATERIALS AND METHODS}

Permission was obtained from the Jawaharlal Institute of Postgraduate Medical Education and Research Ethical Committee, Human Studies (Reg. No. JIP/IEC/2018/079). Ethical issues involved were minimal. Informed consent was obtained from every participant's parent/legally authorized/acceptable representative since they could not provide consent because of critical illness and inability/unconsciousness after a brief explanation regarding the study by the investigator. Confidentiality of the data, the right to withdraw from the study, and anonymity of the subjects were explained before data collection. Good Clinical Practice guidelines of the Indian Council for Medical Research were followed. Patient data were stored confidentially.

Prospective cross-sectional audit of intubated and mechanically ventilated patients in ICUs of a tertiary care hospital was performed. This audit was carried out every three days in 11 ICUs of a tertiary care hospital between August 2019 to November 2019 in patients who were intubated with a cuffed endotracheal tube (ETT) and mechanically ventilated in the

\section{KEYMESSAGES}

- This study demonstrated that intensive care units intensive care units (ICUs) with cuff pressure monitoring devices and active measurement protocols had a higher proportion of patients with optimal cuff pressure (58.5\%) and a lower proportion with sub-optimal and high cuff pressures (19.5\% and $22.0 \%$ ) compared to ICUs with no device-based monitoring protocols.

- The ventilator-associated pneumonia rates of ICUs exhibited a weak positive correlation with sub-optimal cuff pressure.

ICUs on the day of the visit. The date and time of the visits were determined randomly using a computer spreadsheet program so that any changes in practice (that is, adjusting the cuff pressure in anticipation of the visit) caused by the study were minimized. The study excluded patients intubated with non-air cuffed ETTs where pressure cannot be measured and non-invasively ventilated patients without ETT.

The device that was used for sample collection was a COVIDIEN-Shiley Hi-Lo Hand pressure Gauge (VBM Medizintechnik, Sulz am Neckar, Germany). Cuff pressure readings were obtained by connecting the pilot balloon to the cuff pressure manometer. The normal pressure range of $20-30 \mathrm{~cm} \mathrm{H}_{2} \mathrm{O}$ was considered optimal cuff pressure, $<20 \mathrm{~cm} \mathrm{H}_{2} \mathrm{O}$ was sub-optimal, and high cuff pressure was $>30 \mathrm{~cm} \mathrm{H}_{2} \mathrm{O}$. Exact readings were documented in an online data entry sheet (google sheet). When the investigator observed that the pressure was too high or too low, it was immediately corrected using the same device, or the assigned nursing personnel was notified to correct it since it is unethical to leave the cuff pressure at suboptimal level. Multiple measurements were performed from a single patient if they continued to be on a ventilator for a longer period. These additional measurements did not affect the study since the investigators were only studying the proportion of instances of the cuff pressure measurements of beds in an ICU where the cuff pressures and VAP rates were normal according to the number of hours of ventilation days rather than per patient.

The institute has a dedicated team of infection control nurses who collect VAP rates based on everyday clinical assessments with updated Centers for Disease Control and Prevention diagnostic criteria. The investigators accessed VAP rate data per month from the infection control department. These rates were calculated every month, and data of a particular month were used when the investigator had performed cuff 
pressure measurements. Since the measurements were performed for 4 months, investigators calculated the average of the VAP rates and correlated it with cuff pressure.

A primary survey was conducted every month in the ICUs during the course of the study to determine whether the ICUs were using a cuff pressure monitor or if one was available and if there was an active usage protocol. The study also surveyed some of the other confounding factors that can affect VAP rates like head end elevation, availability of bed side hand rubbing, closed suctioning system availability, and nurse to patient ratio in the unit at the time of the survey.

Statistical analysis was carried out using the IBM SPSS ver. 22 (IBM SPSS Inc., Armonk, NY, USA). Frequencies and percentages were used for cuff pressure assessment, and proportions were used to describe the number of patients who had optimal cuff pressure. The Kruskal-Wallis test, Spearman rank correlation, chi-square test, Mann-Whitney test, and Fisher's exact test were used to summarize the data. All the statistical tests were carried out at a 5\% level of significance, and a P-value less than 0.05 was considered significant.

\section{RESULTS}

The survey obtained 348 discreet cuff pressure readings from mechanically ventilated patients from 11 ICUs. The ICUs that had a smaller number of cuff pressure readings were combined for interpretation (ICUs that contributed less than 15 cuff pressure readings combined were obstetrics and gynecology ICU $[n=2]$, plastic surgery ICU $[n=9]$, respiratory care center ICU [n=1], surgical ICU [n=14], and urology ICU [n=3]). Among the 348 cuff pressure readings obtained, the majority of the readings were in the high cuff pressure category at
$140(40.2 \%)$, optimum cuff pressure was at $123(35.3 \%)$, and sub-optimal cuff pressure was at 85 (25\%).

Table 1 indicates that the most frequent sub-optimal cuff pressure readings were observed in the neuromedicine ICU at $42.1 \%(n=16)$ and the fewest were noted in the emergency medical services high dependency unit at $15.6 \%(n=10)$. High cuff pressure readings were more prominent in the survey where most of the cuff pressure readings were obtained from the emergency medical services (EMS) high dependency unit (HDU) at 59.4\% ( $\mathrm{n}=38)$. A smaller number of high cuff pressure readings was found in the neurotrauma ICU, at $16.7 \%(n=10)$. The maximum number of optimal-cuff pressure readings were obtained from the Neurotrauma ICU at $65 \%(n=39)$, and the minimum was recorded in the neuro-medicine ICU at $21.1 \%$ $(\mathrm{n}=8)$.

Another result of the audit showed that only two of the total 11 ICUs that participated had a dedicated device for monitoring and an active measurement protocol (AMP). Therefore, $76.44 \%(n=266)$ cuff pressure readings were obtained from ICUs where there was no device for monitoring and no AMP. Only $23.56 \%$ ( $n=82)$ were obtained from ICUs that had dedicated equipment and AMP for monitoring. These findings were reflected in the cuff pressure distribution among the categories, as depicted in Table 2, which shows a significant association between AMP and cuff pressure category $(\mathrm{P}=0.001)$. ICUs with monitoring had lower proportions of sub-optimal (19.5\%) and high (22.0\%) cuff pressure compared to those without AMP (25.9\% and 45.9\%, respectively). The proportion of optimal cuff pressure (58.5\%) was greater in ICUs with AMP compared to those that did not $(28.2 \%)$. Our results showed a weak positive correlation of sub-optimal cuff pressure with VAP rate, as depicted in Table 3 . In addition, we identified

Table 1. Frequency distribution of CP among ICU

\begin{tabular}{|c|c|c|c|}
\hline ICU & Sub-optimal $\mathrm{CP}\left(<20 \mathrm{~cm} \mathrm{H}_{2} \mathrm{O}\right)$ & Optimal CP $\left(20-30 \mathrm{~cm} \mathrm{H}_{2} \mathrm{O}\right)$ & High $\mathrm{CP}\left(>30 \mathrm{~cm} \mathrm{H}_{2} \mathrm{O}\right)$ \\
\hline CTVS ICU $(n=49)$ & $16(32.7)$ & $16(32.4)$ & $17(34.7)$ \\
\hline EMS HDU $(n=64)$ & $10(15.6)$ & $16(25.0)$ & $38(59.4)$ \\
\hline EMS ICU (n=83) & $15(18.1)$ & $25(30.1)$ & 43 (51.8) \\
\hline Neuromedicine ICU ( $n=38)$ & $16(42.1)$ & 8 (21.1) & $14(36.8)$ \\
\hline Neurosurgery ICU (n=25) & $5(20.0)$ & $11(44.0)$ & $9(36)$ \\
\hline Neuro trauma ICU $(n=60)$ & $11(18.3)$ & $39(65.0)$ & $10(16.7)$ \\
\hline Other ICUs $(n=29)^{a}$ & $12(41.3)$ & 8 (27.58) & $9(31.0)$ \\
\hline Total $(n=348)$ & $85(24.4)$ & $123(35.3)$ & $140(40.2)$ \\
\hline
\end{tabular}

Values are presented as number (\%). Total number of cuff pressure measurement=348.

ICU: intensive care unit; CP: cuff pressure; CTVS: cardio thoracic and vascular surgery; EMS: emergency medical services; HDU: high dependency unit.

${ }^{a}$ ICUs where less than 15 cuff pressure measurements were made and those ICUs are the following respective: ICU, surgical ICU, obstetrics and gynaecology ICU, plastic surgery ICU, and urology ICU. 
Table 2. Association of CP monitor availability and active measurement protocol with cuff pressure category

\begin{tabular}{|c|c|c|c|c|}
\hline \multirow[b]{2}{*}{ Group } & \multicolumn{3}{|c|}{$\mathrm{CP}$ category } & \multirow[b]{2}{*}{ P-value } \\
\hline & $\begin{array}{c}\text { Sub-optimal CP } \\
\left(<2 \mathrm{~cm} \mathrm{H}_{2} \mathrm{O}, \mathrm{n}=85\right)\end{array}$ & $\begin{array}{c}\text { Optimal CP } \\
\left(20-30 \mathrm{~cm} \mathrm{H}_{2} \mathrm{O}, \mathrm{n}=123\right)\end{array}$ & $\begin{array}{c}\text { High CP } \\
\left(>30 \mathrm{~cm} \mathrm{H}_{2} \mathrm{O}, \mathrm{n}=140\right)\end{array}$ & \\
\hline Cuff pressure monitor \& active measurement protocol & & & & $0.001^{b}$ \\
\hline No $(n=266)$ & $69(25.9)$ & $75(28.2)$ & $122(45.9)$ & \\
\hline Yes $(n=82)$ & $16(19.5)$ & $48(58.5)$ & $18(22.0)$ & \\
\hline
\end{tabular}

Values are presented as number (\%).

$\mathrm{CP}$ : cuff pressure.

${ }^{a}$ Chi-square test; ${ }^{b} \mathrm{P}<0.05$.

Table 3. Correlation of VAP rate with cuff P category among mechanically ventilated patients in ICUs where the P stands for pressure

\begin{tabular}{lrrc}
\hline Variable & No. $(\%)$ & \multicolumn{1}{c}{$r^{a}$} & P-value \\
\hline Sub-optimal $\mathrm{CP}\left(<20 \mathrm{~cm} \mathrm{H}_{2} \mathrm{O}\right)$ & $85(24.4)$ & 0.214 & $0.04^{\mathrm{b}}$ \\
Optimal CP $\left(2 \mathrm{O}-30 \mathrm{~cm} \mathrm{H}_{2} \mathrm{O}\right)$ & $123(35.3)$ & -0.076 & 0.40 \\
High CP $\left(>30 \mathrm{~cm} \mathrm{H}_{2} \mathrm{O}\right)$ & $140(40.2)$ & 0.109 & 0.20
\end{tabular}

VAP: ventilator-associated pneumonia; ICU: intensive care unit; $\mathrm{CP}$ : cuff pressure.

${ }^{a}$ Spearman rank correlation coefficient; ${ }^{b} \mathrm{P}<0.05$.

some confounding factors of VAP, as provided in Table 4.

\section{DISCUSSION}

The present study showed that most of the cuff pressure values were high, at a percentage of $40.2 \%(n=140)$, while optimal cuff and sub-optimal cuff pressures were found in $35.3 \%$ and $24.4 \%$ of patients, respectively. A high cuff pressure can compromise perfusion and cause impairments in the wall of the trachea and adjacent anatomical structures $[5,8,9]$. Therefore, need for an effective cuff pressure measuring protocol and device-based monitoring is high.

A cuff pressure greater than $30 \mathrm{~cm} \mathrm{H}_{2} \mathrm{O}$ is enough to compromise the anterolateral trachea, affect microcirculation, and lead to multiple complications such as sore throat, hoarseness, stenosis of the trachea, rupture of the trachea, injury, and tracheal esophageal fistula [6,10-12]. Several studies surveyed endo-tracheal tube cuff pressure monitoring and found a similar higher incidence of high cuff pressure in emergency departments $[13,14]$.

Our results also showed that a cuff pressure monitoring device and protocol significantly increased the proportion of patients with optimal cuff pressure and reducing the sub-optimal cuff pressure to $19.5 \%$ and high cuff pressure $22.0 \%$ when compared to those ICU's didn't which did not have the same reported the proportion of sub-optimal cuff pressure as $25.9 \%$, High cuff pressure was $45.9 \%$ and Optimal cuff pres-
Table 4. Distribution of confounding factors for ventilator associated pneumonia during cuff pressure measurements

\begin{tabular}{lr}
\hline Variable & No. $(\%)$ \\
\hline Availability of closed suction among cuff pressure measured & \\
No & $327(94)$ \\
Yes & $21(6.0)$ \\
Hand rub availability & \\
No & $96(27.6)$ \\
Yes & $252(72.4)$ \\
Nurse patient ratio & \\
$1: 2$ & $5(1.4)$ \\
$1: 3$ & $277(79.6)$ \\
$1: 5$ & $6(1.7)$ \\
$1: 6$ & $2(0.6)$ \\
$1: 10$ & $58(16.7)$ \\
Head end elevation & $134(38.5)$ \\
Low (head elevation $\left.<30^{\circ}\right)$ & $197(56.6)$ \\
Normal (head elevation -30 to $\left.-45^{\circ}\right)$ & $17(4.9)$ \\
High (head elevation $\left.>45^{\circ}\right)$ &
\end{tabular}

sure was $28.2 \%$ which was significant $(\mathrm{P}<0.001)$. A previous quality enhancement study reported concurrent results when using cuff pressure manometers [15]. The cuff pressure range was significant $(\mathrm{P}=0.0003)$, and an increase in the safe range of cuff pressure was observed after the introduction of cuff pressure monitoring, AMP, and departmental education. In addition, before implementation, most of the cuff pressure values were high.

There are inadequate data to guide clinicians on the optimal frequency of ETT cuff pressure measurements. Current practices differ throughout the world, from very infrequent to continuous assessments of cuff pressure. However, use of a continuous ETT cuff-pressure control system is associated with significantly lower risk of VAP as it lowers the risk of advancement of subglottic secretions into the lower respiratory tract $[16,17]$. 
Temporary drops in endotracheal cuff pressure are important for entry of upper airway secretions into the lower airways and increase in the incidence of VAP. Studies have shown that continuous pressure control and optimal ET cuff pressure minimize leakage of collected secretions that have pooled above the cuff [18]. In this study, we observed a significantly weak positive correlation ( $\mathrm{r}=0.214, \mathrm{P}=0.04)$ of sub-optimal cuff pressure with VAP rate. This can be considered a limitation of the study but without clinical significance. Multivariate analysis performed by Rello et al. [6] also showed a trend toward higher risk of VAP among patients with sub-optimal cuff pressure (relative risk, 2.57 ; 95\% confidence interval, 0.78-8.03).

Furthermore, no continuous data on cuff pressure were maintained to ascertain the relationship, as the primary aim of the study was to establish the relationship between optimal cuff pressure and availability of cuff pressure measurement and AMP [3-5,19].

The main limitation of the present study was that there was no continuous monitoring of cuff pressure and factors that contributed to cuff pressure changes. The study was performed based on instances of measurement and not on individual patients. As a result, the clinical covariates of patients were not considered. This restricted further statistical analysis on the predictors of cuff-pressure variations. Due to the time limitation of the study, unit-wise monthly VAP rate was used for assessing the correlation with cuff pressure since each individual could not be followed-up for the same amount of time. Another limitation was that it was not possible to detect pressure leaks while attaching the device to the pilot balloon during monitoring.

This study suggests provision of cuff pressure monitoring devices for every critical care unit and development of AMP from an administrative level to promote optimal cuff pressure. This will reduce the overall VAP rates in hospitals. Adherence to the protocol can be added as a quality indicator hospital infection control.

\section{CONFLICT OF INTEREST}

No potential conflict of interest relevant to this article was reported.

\section{ORCID}

Biju Viswambharan https://orcid.org/0000-0003-2769-0382
Manjini Jeyaram Kumari

https://orcid.org/0000-0003-4403-300X

Gopala Krishnan https://orcid.org/0000-0003-0458-3883

Lakshmi Ramamoorthy https://orcid.org/0000-0003-4248-1407

\section{AUTHOR CONTRIBUTIONS}

Conceptualization: BV, MJK, GK. Data curation: BV. Formal analysis: BV, MJK, GK. Methodology: MJK, GK. Project administration: BV, MJK, GK. Visualization: all authors. Writing-original draft: BV, MJK, GK. Writing-review \& editing: all authors.

\section{REFERENCES}

1. Magill SS, O'Leary E, Janelle SJ, Thompson DL, Dumyati G, Nadle J, et al. Changes in prevalence of health care-associated infections in U.S. hospitals. N Engl J Med 2018;379:1732-44.

2. Haas CF, Eakin RM, Konkle MA, Blank R. Endotracheal tubes: old and new. Respir Care 2014;59:933-52.

3. Letvin A, Kremer P, Silver PC, Samih N, Reed-Watts P, Kollef $\mathrm{MH}$. Frequent versus infrequent monitoring of endotracheal tube cuff pressures. Respir Care 2018;63:495-501.

4. Akdogan O, Ersoy Y, Kuzucu C, Gedik E, Togal T, Yetkin F. Assessment of the effectiveness of a ventilator associated pneumonia prevention bundle that contains endotracheal tube with subglottic drainage and cuff pressure monitorization. Braz J Infect Dis 2017;21:276-81.

5. Hamilton VA, Grap MJ. The role of the endotracheal tube cuff in microaspiration. Heart Lung 2012;41:167-72.

6. Rello J, Soñora R, Jubert P, Artigas A, Rué M, Vallés J. Pneumonia in intubated patients: role of respiratory airway care. Am J Respir Crit Care Med 1996;154:111-5.

7. Álvarez-Lerma F, Sánchez García M; Task Force of Experts for Project "Zero VAP" in Spain. "The multimodal approach for ventilator-associated pneumonia prevention"-requirements for nationwide implementation. Ann Transl Med 2018;6:420.

8. Fu Y, Xi X. Analysis on risk factors of endotracheal cuff under inflation in mechanically ventilated patients. Zhonghua Wei Zhong Bing Ji Jiu Yi Xue 2014;26:870-4.

9. Nseir S, Brisson H, Marquette CH, Chaud P, Di Pompeo C, Diarra M, et al. Variations in endotracheal cuff pressure in intubated critically ill patients: prevalence and risk factors. Eur J Anaesthesiol 2009;26:229-34.

10. Nseir S, Zerimech F, Jaillette E, Artru F, Balduyck M. Microaspiration in intubated critically ill patients: diagnosis and prevention. Infect Disord Drug Targets 2011;11:413-23. 
11. Jaillette E, Zerimech F, De Jonckheere J, Makris D, Balduyck M, Durocher A, et al. Efficiency of a pneumatic device in controlling cuff pressure of polyurethane-cuffed tracheal tubes: a randomized controlled study. BMC Anesthesiol 2013;13:50.

12. Sengupta P, Sessler DI, Maglinger P, Wells S, Vogt A, Durrani J, et al. Endotracheal tube cuff pressure in three hospitals, and the volume required to produce an appropriate cuff pressure. BMC Anesthesiol 2004;4:8.

13. Ranjan N, Chaudhary U, Chaudhry D, Ranjan KP. Ventilator-associated pneumonia in a tertiary care intensive care unit: analysis of incidence, risk factors and mortality. Indian J Crit Care Med 2014;18:200-4.

14. Mukhopadhyay C, Bhargava A, Ayyagari A. Role of mechanical ventilation \& development of multidrug resistant organisms in hospital acquired pneumonia. Indian J Med Res 2003;118:22935.

15. Stevens GJ, Warfel JW, Aden JK, Blackwell SD. Intraoperative endotracheal cuff pressure study: how education and availability of manometers help guide safer pressures. Mil Med 2018;183:e416-9.

16. Rouzé A, Martin-Loeches I, Nseir S. Airway devices in ventilator-associated pneumonia pathogenesis and prevention. Clin Chest Med 2018;39:775-83.

17. Jaillette E, Girault C, Brunin G, Zerimech F, Behal H, Chiche A, et al. Impact of tapered-cuff tracheal tube on microaspiration of gastric contents in intubated critically ill patients: a multicenter cluster-randomized cross-over controlled trial. Intensive Care Med 2017;43:1562-71.

18. Alzahrani AR, Al Abbasi S, Abahoussin OK, Al Shehri TO, Al-Dorzi HM, Tamim HM, et al. Prevalence and predictors of out-of-range cuff pressure of endotracheal and tracheostomy tubes: a prospective cohort study in mechanically ventilated patients. BMC Anesthesiol 2015;15:147.

19. Huang WM, Huang XA, Du YP, Li LX, Wu FF, Hong SQ, et al. Tapered cuff versus conventional cuff for ventilator-associated pneumonia in ventilated patients: a meta-analysis of randomized controlled trials. Can Respir J 2019;2019:7876417. 